\title{
Mass and Spin of Double Dual Solutions in Poincaré Gauge Theory.
}

E. W. Mielke $\left(^{*}\right)$ and R. P. Wallner $(* *)$

Institute for Theoretical Physics, University of Cologne

D-5000 Köln 41, Federal Republic of Germany

(Nuovo Cimento B, 101, 607 (1988))

Equations (3.10) and (5.3), respectively, should read

$$
\begin{aligned}
& \gamma\left[\frac{1}{2} R_{\psi k \nu}^{\{\}} \wedge *\left(\vartheta^{\mu} \wedge \mho^{*} \wedge \vartheta_{x}\right)-\Lambda_{\text {eff }} * \vartheta_{x}\right]=l^{2} \tilde{\Sigma}_{x}, \\
& \underline{R}^{x^{\beta}}=-\frac{\xi}{2 N} \stackrel{*}{ } R_{\perp \gamma^{\circ}} \epsilon^{x \beta \gamma^{j}}-\gamma \frac{x}{l^{2}} \underline{\vartheta}^{x} \Lambda \underline{\vartheta}^{\beta},
\end{aligned}
$$

whereas the completed reference $\left({ }^{20}\right)$ reads

P. Baeckler and E. W. Mielke: Fortschr. Phys., 36, 549 (1988). 